\title{
Isolation and characterization of microsatellite markers for Osmanthus fragrans (Oleaceae) using 454 sequencing technology
}

\author{
W.J. Yuan ${ }^{1 *}$, Y. Li2 ${ }^{2 *}$, Y.F. $\mathrm{Ma}^{3}$, Y.J. $\mathrm{Han}^{3}$ and F.D. Shang ${ }^{3}$ \\ ${ }^{1}$ Institute of Chinese Materia Medica, Henan University, Kaifeng, China \\ ${ }^{2}$ College of Forestry, Henan Agricultural University, Zhengzhou, China \\ ${ }^{3}$ College of Life Sciences, Henan University, Kaifeng, China \\ *These authors contributed equally to this study. \\ Corresponding author: F.D. Shang \\ E-mail: liyongrui1@126.com \\ Genet. Mol. Res. 14 (4): 17154-17158 (2015) \\ Received August 25, 2015 \\ Accepted October 18, 2015 \\ Published December 16, 2015 \\ DOI http://dx.doi.org/10.4238/2015.December.16.15
}

ABSTRACT. Osmanthus fragrans (Oleaceae) is an evergreen shrub or small tree that grows in south China. In this study, Roche $454 \mathrm{FLX}+$ sequencing combined with the magnetic bead enrichment method was used to isolate microsatellite markers from the genome of $O$. fragrans. A total of 1471 microsatellites that contained enough flanking sequences for primer pair design were identified from 89,633 raw sequencing reads. One hundred primer pairs were randomly chosen to test primer amplification efficiency. Among these tested primer pairs, 20 yielded polymorphic amplification products across 16 individuals from the Albus, Luteus, and Aurantiacus groups. The number of alleles ranged from 2 to 6 , with an average of 3.7. The observed heterozygosity ranged from 0 to 0.813 , with an average of 0.460 . Shannon's information index ranged from 0.463 to 1.707, with an average of 0.975 . Six loci (Of 05, Of 06, Of 08, Of 12, Of 15 , and $O f 19$ ) deviated significantly from Hardy-Weinberg equilibrium ( $P$ $<0.05$ ), which was due to an excess of homozygotes or heterozygotes. Nine pairs of loci (Of 01 and Of 05; Of 04 and Of 05; Of 01 and Of 06; Of 
04 and Of 12; Of 02 and Of 13; Of 04 and Of 13; Of 12 and Of 13; Of 04 and $O f$ 19; Of 05 and of 19) showed significant linkage disequilibrium, which indicated significant allelic association between the loci. This set of microsatellite markers will be valuable for molecular marker-assisted breeding in $\mathrm{O}$. fragrans.

Key words: Molecular marker-assisted breeding; Microsatellite markers; Osmanthus fragrans; Roche 454 sequencing

\section{INTRODUCTION}

Osmanthus fragrans (Oleaceae) is an evergreen shrub or small tree that grows in south China (Xu et al., 2014). As an ornamental plant, O. fragrans is renowned for its attractive and sweet fragrance, and is regarded as one of the top ten Chinese traditional flowers. This species has been cultivated for more than 2500 years in China. A total of 166 cultivars have been identified, belonging to four groups: Asiaticus, Albus, Luteus, and Aurantiacus (Xiang and Liu, 2008). To date, a few studies have reported on the genetic diversity of $O$. fragrans cultivars using amplified fragment length polymorphism (AFLP; Han et al., 2008; Yan et al., 2009; Yuan et al., 2011), sequencerelated amplified polymorphism (SRAP; Li et al., 2009), microsatellite (Zhang et al., 2011; Duan et al., 2013), and inter-simple sequence repeat (ISSR; Fan et al., 2014) markers. However, AFLPs, SRAPs, and ISSRs are dominant markers, which pose difficulties for heterozygosity calculations and paternity analysis. Microsatellites are a type of powerful marker used in molecular markerassisted breeding for their high polymorphism, ease of genotyping, and co-dominant inheritance (Oliveira et al., 2006). However, the limited number of microsatellite markers for O. fragrans (Zhang et al., 2011; Duan et al., 2013) fails to meet the high marker density required in molecular breeding. Thus, it is necessary to develop a set of more powerful markers for molecular marker-assisted breeding in this famous ornamental plant. Next generation sequencing now allows us to obtain a large number of markers in a short time. In this study, we utilized Roche 454 sequencing technology to isolate microsatellite markers for $O$. fragrans.

\section{MATERIAL AND METHODS}

\section{Isolation of microsatellite markers}

Genomic DNA was extracted from fresh leaves of $O$. fragrans using a plant genomic DNA isolation kit (Tiangen Biotech, Beijing, China), according to the manufacturer protocol. Approximately $2 \mu \mathrm{g}$ genomic DNA was used to generate a shotgun library, following the 454 Roche protocol. Hybridization of eight 5'-biotinylated oligonucleotides [(AG)10, (AC)10, (AAC)8, (ACG)8, (AAG)8, (ACAT)6, (ATCT)6, and (AGG)8] with the shotgun library was performed to enrich the repetitive motifs. The enriched products were subsequently sequenced using a Roche 454 FLX+ sequencing platform (Roche Applied Science, Indianapolis, IN, USA). Microsatellite identification was performed using MISA (Thiel et al., 2003) with a minimum of five repeats for di-, tri-, tetra-, penta-, and hexanucleotide motifs. Primer pairs were designed using Primer3 (Rozen and Skaletsky, 2000), with default parameters. 


\section{Polymerase chain reaction (PCR) amplification and genotyping}

Sixteen individuals of $O$. fragrans were collected from the Albus (6 individuals), Luteus (5 individuals), and Aurantiacus groups (5 individuals) in Henan University (Kaifeng, China). PCR amplifications of each sample were performed in a $20-\mu \mathrm{L}$ reaction volume containing 50 ng genomic DNA, $0.5 \mu \mathrm{M}$ each primer, and $10 \mu \mathrm{L} 2 \mathrm{X}$ Taq PCR MasterMix (0.1 U Taq polymerase/ $\mu \mathrm{L}, 0.5 \mathrm{mM}$ dNTPs, 20 mM Tris-HCl, pH 8.3) using an S1000 Thermal cycler (Bio-Rad, USA). PCR amplification conditions were set as follows: pre-incubation at $94^{\circ} \mathrm{C}$ for $5 \mathrm{~min}$; followed by 35 cycles of denaturation at $94^{\circ} \mathrm{C}$ for $50 \mathrm{~s}$; annealing at a locus-specific annealing temperature for $45 \mathrm{~s}$; elongation at $72^{\circ} \mathrm{C}$ for $50 \mathrm{~s}$; and a final extension step at $72^{\circ} \mathrm{C}$ for $8 \mathrm{~min}$. PCR product $(5 \mu \mathrm{L})$ was mixed with $1 \mu \mathrm{L}$ $6 \mathrm{X}$ loading buffer and then electrophoresed on $8 \%$ native polyacrylamide gels. PCR products were visualized using silver staining, and band sizes were reported using a 50 bp DNA ladder (Takara, Dalian, Liaoning, China) as a reference.

\section{Data analysis}

Electropherograms for the fragment analysis were analyzed using GeneMarker v1.95 (SoftGenetics, State College, Pennsylvania, USA). The number of alleles per locus $\left(N_{\mathrm{A}}\right)$, observed heterozygosity $\left(H_{\mathrm{O}}\right)$, expected heterozygosity $\left(H_{\mathrm{E}}\right)$, and Shannon's information index (SII) were calculated using Popgene 1.32 (Yeh et al., 1997). Linkage disequilibrium (LD) and Hardy-Weinberg equilibrium (HWE) were tested using Fisher's exact tests using GENEPOP 4.2 (Rousset, 2008).

\section{RESULTS AND DISCUSSION}

A total of 89,633 raw sequencing reads were yielded using Roche 454 sequencing. These reads ranged from 90 to $589 \mathrm{bp}$, with an average length of $192 \mathrm{bp}$. Of these, 1471 sequences were found to contain microsatellites that contained enough flanking sequences for primer pair design and were deposited in GenBank (accession No. KP031709-312207, KP179450-180421). To test primer amplification efficiency, 100 sequences were randomly chosen to design primer pairs, and PCRs were performed. Of the 100 primer pairs, 72 were abandoned because of unsuccessful amplification and non-target amplification, whereas the remaining 28 primer pairs were tested for polymorphisms across 16 individuals from the Albus, Luteus, and Aurantiacus groups.

A total of 20 primer pairs yielded polymorphic amplification products (Table 1). The genetic diversity parameters of each group are presented in Table 2. $N_{\mathrm{A}}$ ranged from 2 (Of 06 , Of 18, and Of 20) to 6 (Of 05, Of 08, and Of 13), with an average of 3.7. $H_{0}$ ranged from 0 (Of 06) to 0.813 (Of 11), with an average of 0.460. SII ranged from 0.463 (Of 09) to 1.707 (Of 08), with an average of 0.975. Six loci (Of 05, Of 06, Of 08, Of 12, Of 15, and Of 19) deviated significantly from HWE (P < 0.05 ), which was due to an excess of homozygotes or heterozygotes. Nine pairs of loci (Of 01 and Of 05; Of 04 and Of 05; Of 01 and Of 06; Of 04 and Of 12; Of 02 and Of 13; Of 04 and Of 13; Of 12 and Of 13; Of 04 and Of 19; Of 05 and Of 19) showed significant LD, which indicated significant allelic association between the loci. This set of microsatellite markers has increased the marker density of the $O$. fragrans genome, which will be valuable for molecular marker-assisted breeding of this species. 
Table 1. Primer sequences and characterization of 20 microsatellite loci isolated from Osmanthus fragrans.

\begin{tabular}{|c|c|c|c|c|c|}
\hline Primer & Primer sequence $\left(5^{\prime}-3^{\prime}\right)$ & Repeat motif & $\mathrm{Ta}\left({ }^{\circ} \mathrm{C}\right)$ & Allele Size (bp) & GenBank accession No. \\
\hline \multirow[t]{2}{*}{ Of01 } & F: ATCCACTCTTCCGATGGTCA & & & & \\
\hline & R: CTGTTTGACGATTTCCGGTT & $($ AAAAAT) 5 & 58 & $347-359$ & KP031709 \\
\hline \multirow[t]{2}{*}{ Of02 } & F: AAACAGGAACCACAAAACCG & & & & \\
\hline & R: CCAATCCCATGCTTGAGTCT & $(A G) 15$ & 58 & $282-286$ & KP031710 \\
\hline \multirow[t]{2}{*}{ Of03 } & F: AAGGGGCCTAGGATCGTTTA & & & & \\
\hline & R: GTGAGATGGCACCGTAGGAT & $(A G G) 11$ & 58 & $284-293$ & KP031711 \\
\hline \multirow[t]{2}{*}{ Of04 } & F: CGACGACGCTTACCCTTATT & & & & \\
\hline & R: CCATTTCTCGCCTATCAAGG & (ATAC) 11 & 58 & 291-299 & KP031712 \\
\hline \multirow[t]{2}{*}{ Of05 } & F: AAATCCCAACTCACAAGGCA & & & & \\
\hline & R: TCTGCTGCACTCTTCATTGC & (CA) 13 & 58 & $208-218$ & KP031713 \\
\hline \multirow[t]{2}{*}{ Of06 } & F: AAATGGGTGACTGGGAGTTG & & & & \\
\hline & R: TGGGCCCTTTTCTGTTCTTA & (GT)8(GA)15(GT)8 & 58 & $339-342$ & KP031714 \\
\hline \multirow[t]{2}{*}{ Of07 } & F: GCAAAAATGTGAAACGACGA & & & & \\
\hline & R: TGGCATGTATTCCAGGTTCA & (GTGA)9 & 60 & 283-291 & KP031715 \\
\hline \multirow[t]{2}{*}{ Of08 } & F: TGTTGTAACCGCCTTCCAAT & & & & \\
\hline & R: AGAAACTCCAGGGGCTGATT & (TA)11 & 60 & $309-319$ & KP031716 \\
\hline \multirow[t]{2}{*}{ Ofo9 } & F: TTGCCACTAGTTTTCACCCC & & & & \\
\hline & R: TTGGGGCCACTAGAAACAAC & (TA)8(TG)17 & 58 & $240-246$ & KP031717 \\
\hline \multirow[t]{2}{*}{ Of10 } & F: TTGGTGACACAATCGACAAT & & & & \\
\hline & R: AAGGGCAACAGCTTCTGAAA & (TC)6(AC) 14 & 58 & $335-347$ & KP031718 \\
\hline \multirow[t]{2}{*}{ Of11 } & F: CCGCATTAACACCCAAACTT & & & & \\
\hline & R: CAAAAGAAGACCAATCCCGA & (TC) $9(\mathrm{CA}) 23$ & 58 & $395-401$ & KP031719 \\
\hline \multirow[t]{2}{*}{ Of12 } & F: AATGTGTCATTCAAGGGGGA & & & & \\
\hline & R: ATGCCTTGTTTGGCTCTCAT & (TCT) 14 & 58 & $304-312$ & KP031720 \\
\hline \multirow[t]{2}{*}{ Of13 } & F: CCATTTTGGTCTTCTCCCAA & & & & \\
\hline & R: CCAATGAAAAGCCACCATCT & (TCT)5C(CTT) 6 & 58 & $388-402$ & KP031721 \\
\hline \multirow[t]{2}{*}{ Of14 } & F: TGGTCCGCTAAATAACCGAG & & & & \\
\hline & R: TGGGAGCTCTCCTTCAACAT & (CTT)13 & 58 & 313-322 & KP031722 \\
\hline \multirow[t]{2}{*}{ Of15 } & F: CTGAAGCCCGATCCATTAAC & & & & \\
\hline & R: GCTAGGGCACATATCACCGT & (GT)8(GA)17 & 60 & $331-337$ & KP031723 \\
\hline \multirow[t]{2}{*}{ Of16 } & F: TGCCACTACCATTTTCACCA & & & & \\
\hline & R: TCAAACTCCTTGCCACAAAA & (TG)12 & 60 & $241-247$ & KP031724 \\
\hline \multirow[t]{2}{*}{ Of17 } & F: GGGTGTGCTGTGTTGATGTC & & & & \\
\hline & R: CСССТСТСАAАTTGCAAGAA & (TG)17(AG)11 & 60 & $331-335$ & KP031725 \\
\hline \multirow[t]{2}{*}{ Of18 } & F: TTTTGTTTGGCCTTTCTTGG & & & & \\
\hline & R: AGATCATCGGACGGGTACAG & (TG)21 & 60 & $381-383$ & KP031726 \\
\hline \multirow[t]{2}{*}{ Of19 } & F: GCATTGCGGATTTTTCATCT & & & & \\
\hline & R: TTGGGGTATCCAAATCCGTA & (TG)7(GA)15 & 60 & $238-244$ & KP031727 \\
\hline \multirow[t]{2}{*}{ Of20 } & F: TCGGCAAAAACTTGCAGAC & & & & \\
\hline & R: ATCCACGTCAATGTGCGATA & (TG)23(AG)9 & 60 & $342-344$ & KP031728 \\
\hline
\end{tabular}

Ta, PCR annealing temperature.

Table 2. Genetic diversity parameters for three groups of Osmanthus fragrans assessed using the 20 newly developed microsatellite loci.

\begin{tabular}{|c|c|c|c|c|c|c|c|c|c|c|c|c|c|}
\hline \multirow[t]{2}{*}{ Primer } & \multicolumn{3}{|c|}{ Albus group $(\mathrm{N}=6)$} & \multicolumn{3}{|c|}{ Luteus group $(\mathrm{N}=5)$} & \multicolumn{3}{|c|}{ Aurantiacus group $(\mathrm{N}=5)$} & \multicolumn{4}{|c|}{ Total $(\mathrm{N}=16)$} \\
\hline & $N_{\mathrm{A}}$ & $H_{\mathrm{E}}$ & $H_{\mathrm{o}}$ & $N_{\mathrm{A}}$ & $H_{\mathrm{E}}$ & $H_{0}$ & $N_{\mathrm{A}}$ & $H_{\mathrm{E}}$ & $H_{\mathrm{o}}$ & $N_{\mathrm{A}}$ & $H_{\mathrm{E}}$ & $H_{0}$ & SII \\
\hline Of01 & 2 & 0.167 & 0.167 & 3 & 0.644 & 0.800 & 2 & 0.200 & 0.200 & 3 & 0.365 & 0.375 & 0.615 \\
\hline OfO2 & 2 & 0.530 & 0.167 & 3 & 0.644 & 0.800 & 2 & 0.467 & 0.200 & 3 & 0.522 & 0.375 & 0.786 \\
\hline Of03 & 3 & 0.682 & 0.833 & 3 & 0.733 & 0.800 & 3 & 0.644 & 0.600 & 3 & 0.679 & 0.750 & 1.086 \\
\hline Of04 & 3 & 0.439 & 0.167 & 2 & 0.467 & 0.600 & 3 & 0.511 & 0.200 & 3 & 0.446 & 0.313 & 0.743 \\
\hline Of05 & 4 & 0.652 & 0.167 & 4 & 0.733 & 0.600 & 3 & 0.511 & 0.400 & 6 & 0.730 & $0.375^{*}$ & 1.444 \\
\hline Of06 & 2 & 0.546 & 0.000 & 2 & 0.356 & 0.000 & 2 & 0.533 & 0.000 & 2 & 0.508 & $0.000^{*}$ & 0.685 \\
\hline Of07 & 3 & 0.667 & 0.333 & 4 & 0.711 & 0.800 & 3 & 0.689 & 0.800 & 4 & 0.700 & 0.625 & 1.237 \\
\hline Of08 & 5 & 0.742 & 0.833 & 4 & 0.711 & 0.400 & 4 & 0.822 & 0.200 & 6 & 0.831 & $0.500^{*}$ & 1.707 \\
\hline Of09 & 3 & 0.439 & 0.167 & 2 & 0.200 & 0.200 & 1 & 0.000 & 0.000 & 3 & 0.234 & 0.125 & 0.463 \\
\hline Of10 & 4 & 0.652 & 0.833 & 2 & 0.467 & 0.600 & 2 & 0.556 & 0.600 & 4 & 0.563 & 0.688 & 0.955 \\
\hline Of11 & 4 & 0.712 & 1.000 & 2 & 0.467 & 0.600 & 4 & 0.644 & 0.800 & 4 & 0.601 & 0.813 & 1.086 \\
\hline Of12 & 3 & 0.667 & 0.167 & 4 & 0.733 & 0.600 & 4 & 0.733 & 1.000 & 5 & 0.702 & $0.750^{*}$ & 1.329 \\
\hline Of13 & 4 & 0.561 & 0.167 & 4 & 0.733 & 0.600 & 4 & 0.733 & 0.800 & 6 & 0.704 & 0.500 & 1.372 \\
\hline Of14 & 3 & 0.318 & 0.333 & 2 & 0.356 & 0.400 & 2 & 0.356 & 0.400 & 4 & 0.337 & 0.375 & 0.672 \\
\hline Of15 & 3 & 0.530 & 0.333 & 3 & 0.511 & 0.200 & 3 & 0.689 & 0.400 & 3 & 0.635 & $0.313^{*}$ & 1.013 \\
\hline Of16 & 2 & 0.409 & 0.500 & 3 & 0.511 & 0.600 & 3 & 0.600 & 0.800 & 4 & 0.478 & 0.625 & 0.821 \\
\hline Of17 & 3 & 0.727 & 0.667 & 3 & 0.511 & 0.600 & 2 & 0.467 & 0.200 & 3 & 0.605 & 0.500 & 0.984 \\
\hline Of18 & 2 & 0.303 & 0.333 & 2 & 0.356 & 0.400 & 2 & 0.355 & 0.400 & 2 & 0.315 & 0.375 & 0.483 \\
\hline Of19 & 3 & 0.621 & 0.667 & 4 & 0.800 & 0.000 & 3 & 0.644 & 0.400 & 4 & 0.748 & $0.375^{*}$ & 1.337 \\
\hline Of20 & 2 & 0.530 & 0.500 & 2 & 0.467 & 0.600 & 2 & 0.467 & 0.200 & 2 & 0.498 & 0.438 & 0.676 \\
\hline
\end{tabular}

$\mathrm{N}$, Number of individuals tested; $N_{\mathrm{A}}$, number of alleles; $H_{\mathrm{E}}$, expected heterozygosity; $H_{\mathrm{O}}$, observed heterozygosity; SII, Shannon's information index; *significant deviation from Hardy-Weinberg equilibrium. 


\title{
Conflicts of interest
}

The authors declare no conflict of interest.

\section{ACKNOWLEDGMENTS}

\author{
Research supported by the National Natural Science Fund in China (\#31270738) and the \\ Henan Province Science and Technology Key Project (\#132102310428).
}

\section{REFERENCES}

Duan YF, Wang XR, Xiang QB, Liang LL, et al. (2013). Genetic diversity of androdioecious Osmanthus fragrans (Oleaceae) cultivars using microsatellite markers. Appl. Plant Sci. 1: 1200092.

Fan FH, Xia H, Wang ZQ and Chen HG (2014). Analysis on the genetic diversity of Osmanthus fragrans Lour. in Xianning with ISSR markers. J. Hubei Univ. 36: 272-276.

Han YJ, Dong MF and Yuan WJ (2008). Study on the genetic diversity of Osmanthus fragrans cultivars. Chin. Bull. Bot. 5: 559-564.

Li M, Hou XL and Hao RM (2009). Analysis of genetic relationships of Osmanthus fragrans based on SRAP markers. Acta Hortic. Sin. 36: 1667-1775.

Oliveira EJ, Padua JG, Zucchi MI, Vencovsky R, et al. (2006). Origin, evolution and genome distribution of microsatellites. Genet. Mol. Biol. 29: 294-307.

Rousset F (2008). Genepop'007: a complete reimplementation of the genepop software for Windows and Linux. Mol. Ecol. Resour. 8: 103-106.

Rozen S and Skaletsky H (2000). Primer3 on the WWW for general users and for biologist programmers. In: Bioinformatics methods and protocols: methods in molecular biology (Misener S and Krawetz S, eds.) Humana Press, Totowa, USA, 365-386.

Thiel T, Michalek W, Varshney RK and Graner A (2003). Exploiting EST database for the development and characterization of gene-derived SSR-markers in barley (Hordeum vulgare L.). Theor. Appl. Genet. 106: 411-422.

Xiang QB and Liu YL (2008). An illustrated monograph of the sweet Osmanthus cultivars in China. Zhejiang Science and Technology Press, Hangzhou, China.

Xu YC, Zhou LH, Hu SQ, Hao RM, et al. (2014). The differentiation and development of pistils of hermaphrodites and pistillodes of males in androdioecious Osmanthus fragrans L. and implications for the evolution to androdioecy. Plant Syst. Evol. 300: 843-849.

Yan XY, Xiao BL, Han YJ, Yuan WJ, et al. (2009). AFLP analysis of genetic relationships and diversity of some Chinese Osmanthus fragrans cultivars. Life Sci. J. 6: 11-16.

Yeh FC, Yang RC, Boyle T, Ye ZH, et al. (1997). POPGENE: the user-friendly shareware for population genetic analysis. Molecular Biology and Biotechnology Centre, University of Alberta, Canada. Available at http://www.ualberta.ca/ fyeh/ popgene.html.

Yuan WJ, Han YJ, Dong MF and Shang FD (2011). Assessment of genetic diversity and relationships among Osmanthus fragrans cultivars using AFLP markers. Electron. J. Biotechnol. 14: 1-9.

Zhang ZR, Fan DM, Guo SQ, Li DZ, et al. (2011). Development of 29 microsatellite markers for Osmanthus fragrans (Oleaceae), a traditional fragrant flowering tree of china. Am. J. Bot. 98: e356-359. 